\section{COVID-19 Pandemic: Are Patients with Inflammatory Bowel Disease Really at Risk?}

Sir,

SARS-COV-2 pandemic has presented with varying clinical manifestations. The United States now accounts for the most number of cases. Initially, COVID-19 manifested as pneumonia of unknown etiology with accompanying symptoms of fever, cough, and mild to severe dyspnea. However, the emergence of gastrointestinal manifestations were noted in a cross-sectional study $(n=204)$, conducted by Pan et al. ${ }^{1}$ Study data reveals that $50 \%(n=103)$ of patients presented with gastrointestinal complaints. ${ }^{1}$ More specifically, symptoms include loss of appetite $(78.6 \%)$, diarrhea (34\%), vomiting (3.9\%), and abdominal pain $(1.9 \%) .{ }^{1}$ The emergence of these gastrointestinal findings has raised serious concerns about the management of patients with a history of digestive problems. This has led to the implementation of several gastrointestinal care recommendations by the Chinese IBD Society, China Crohn's \& Colitis Foundation, Crohn's Colitis of Foundation America, and Crohn's \& Colitis $\mathrm{UK}^{2}{ }^{2}$ The immune system of the gut is the frontline defender against pathogens. It mediates tolerance to both beneficial and pathogenic gut flora. In patients with inflammatory bowel disease (IBD), this homeostasis mechanism seems to be disturbed. ${ }^{2}$ This subsequently triggers a vicious cycle of intestinal epithelial cell inflammation. Therefore, continuous damage leads to a loss of cellular architecture and damages the cellular constituents responsible for maintaining immune tolerance and mucosal immunity.

Recent clinical studies have demonstrated strong interaction between the receptor-binding domain (RBD) of the virus spike protein and cellular angiotensin-converting enzyme 2 (ACE2) receptor. ${ }^{3}$ This suggests that ACE2 receptor-expressing cells could be more infection-prone. It is expressed in pulmonary type 2 alveolar cells, bladder urothelial cells, myocardium, esophagus, and kidney proximal tubules. ${ }^{4}$ However, the highestexpression was noted in the enterocytes of the terminal ileum and colon. Theestablishment of infectionalso depends on the spike protein-mediated fusion of the host cell membrane with the coronavirus envelope. This requires activation of the spike protein by serine proteases. Patients with Crohn's disease tend to have a higher expression of ACE2 receptors when compared to ulcerative colitis. ${ }^{5}$ This is suggestive of ACE2 receptor up-regulation in patients with IBD. Furthermore, from our current understanding, it is well known that up-regulation of various proteases have been implicated in the pathogenesis of IBD. These findings suggest that the gastrointestinal tract can be the optimal site of infection. On the contrary, to date, no such data exists which implicates SARS-CoV-2 infection in IBD patients. ${ }^{2}$ Moreover, no patient with IBD has presented with COVID-19 in three of the largesttertiary IBD centres in Wuhan, China. ${ }^{2}$
These findings can be explained by the existence of different forms of ACE2 receptors. The two different types include a fulllength ACE2 receptor and a soluble ACE2 receptor. Structurally, the full-length is composed of a transmembrane domain and an extracellular domain. ${ }^{5}$ The latter domain is responsible for the binding of the ACE2 receptor to the spike protein of the virus. On the other hand, the soluble type circulates freely and lacks the transmembrane domain. Clinical studies have showed that the soluble ACE2 inhibits SARS-COV replication. ${ }^{4}$ Laboratory studies have also demonstrated that SARS-CoV-2 has a higher affinity for ACE 2 receptors when compared to SARS-CoV. ${ }^{3}$ This higher affinity could suggest that the soluble form might prevent the virus from binding to the membrane-bound full-length ACE2 receptor. Furthermore, Garg et al. demonstrated that patients with IBD have a marked increase in soluble ACE2 and other circulating renin-angiotensin system components. ${ }^{6}$ This, further raises the possibility that the soluble form of ACE2 could diminish the chances of establishing the infection. Overall, these findings do not suggest patients with IBD are at an increased risk of COVID-19.

Many patients with IBD require immunosuppressive medication to maintain remission and prevent disease complications. We strongly advise against the use of medications such as azathioprine, methotrexate, and thiopurines/allopurinol. ${ }^{2}$ These medications have been known to suppress intracellular mechanisms needed to mount a response against pathogens. Using cytokine blockers as an alternative means of therapy could prove to be beneficial in patients with IBD and COVID-19. Common to both conditions are the underlying increase in cytokines and interleukins such as IL-2, IL-6, tumor necrosis factor, and interferon- $\gamma$. They are known to mediate inflammation and damage in both IBD and COVID-19. Therefore, cytokine blockers can help suppress the inflammation that arises as a result of cytokines in IBD. Bearing this in mind, we believe that clinical studies reflecting the possibility of using cytokine blockers to reduce the cytokine storm in COVID-19 patients warrant further exploration. ${ }^{2}$

\section{CONFLICT OF INTEREST:}

Authors declared no conflict of interest.

\section{AUTHORS' CONTRIBUTION:}

$\mathrm{SAH}$, JE: Accessed to the data and played an equal role in writing the manuscript.

\section{REFERENCES}

1. Pan L, Mu M, Ren HG, Yang P, Sun Y, Wang R, et al. Clinical characteristics of covid-19 patients with digestive symptoms in Hubei, China: A descriptive, cross-sectional, multicenter study. Am J Gastroenterol 2020; 115: 10.14309.

2. Mao R, Liang J, Shen J, Ghosh S, Zhu L, Yang H, et al. Implications of COVID-19 for patients with pre-existing digestive diseases. Lan Gastroen Hep 2020; 5(5):425-7.

3. Wrapp D, Wang N, Corbett K, Goldsmith J, Hsieh C, Abiona 
O, et al. Cryo-EM structure of the 2019-nCoV spike in the prefusion conformation. Science 2020; 367(6483): 1260-3.

4. Li W, Moore M, Vasilieva N, Sui J, Wong S, Berne M, et al. Angiotensin-converting enzyme 2 is a functional receptor for the SARS coronavirus. Nature 2003; 426 (6965):450-4.

5. Ning L, Shan G, Sun Z, Zhang F, Xu C, Lou X, et al. Quantitative proteomic analysis reveals the deregulation of nicotinamide adenine dinucleotide metabolism and CD38 in inflammatory bowel disease. Biomed Res Int 2019; 2019:1-11.

6. Garg M, Burrell L, Velkoska E, Griggs K, Angus P, Gibson $P$, et al. Upregulation of circulating components of the alternative renin-angiotensin system in inflammatory bowel disease: A pilot study. J Renin Angiotensin Aldosterone Syst 2014; 16(3):559-69.

Syed Adeel Hassan ${ }^{1}$ and Jude Ezeh

${ }^{1}$ Dow University of Health Sciences, Karachi, Pakistan ${ }^{2}$ Department of Internal Medicine, Newark Beth Israel Medical Center, New Jersey, USA

Correspondence to: Dr. Syed Adeel Hassan, Dow University of Health Sciences, Karachi, Pakistan

E-mail: doc.syed92@gmail.com

Received: June 16, 2020; Revised: June 25, 2020;

Accepted: June 25, 2020

DOI: https://doi.org/10.29271/jcpsp.2020.JCPSPCR.CR79 